\title{
Cost-effective approach to lung cancer risk for a radiological dispersal device (RDD) scenario
}

\author{
Karolina P. S. Costa, \\ Sergio X. Lima, \\ Tercio Brum, \\ Zelmo R. Lima, \\ Jose C. C. Amorim, \\ Matthew J. F. Healy, \\ Helio C. Vital, \\ Matjaž Prah, \\ Edson R. Andrade
}

\begin{abstract}
A release of radioactive material into the environment can lead to hazardous exposure of the population and serious future concerns about health issues such as an increased incidence of cancer. In this context, a practical methodology capable of providing useful basic information from the scenario can be valuable for immediate decisions and future risk assessment. For this work, the simulation of a radiological dispersal device (RDD) filled with americium-241 was considered. The radiation dose simulated by the HotSpot code was used as an input to the epidemiological equations from BEIR $\mathrm{V}$ producing the data used to assess the risk of lung cancer development. The methodology could be useful in providing training for responders aimed to the initial support addressed to decision-making for emergency response at the early phase of an RDD scenario. The results from the simulation allow estimating (a) the size of the potentially affected population, (b) the type of protection action considering gender and location of the individuals, (c) the absorbed doses, (d) the matrix of lung cancer incidence predictions over a period of 5 years, and (e) the cost-effectiveness in the initial decision environment.
\end{abstract}

Keywords: environment $\bullet$ contamination $\bullet$ radiation $\bullet$ cost-effectiveness $\bullet$ risk

K. P. S. Costa, Z. R. Lima

Institute of Nuclear Engineering Graduate Program CNEN, Rio de Janeiro, Brazil

S. X. Lima, T. Brum, J. C. C. Amorim

Defense Engineering Graduate Program

Military Engineering Institute

Rio de Janeiro, Brazil

M. J. F. Healy

Cranfield Forensic Institute, Cranfield University

Defence Academy of the United Kingdom

Shrivenham, SN6 8LA, UK

H. C. Vital

Nuclear Defense Section, IDQBRN/CTEx

Rio de Janeiro, Brazil

M. Prah

Department for Nuclear Power Plant

in Croatian National Electric Utility - HEP d.d.

Zagreb, Croatia

E. R. Andrade ${ }^{凶}$

Institute of Nuclear Engineering Graduate Program

CNEN, Rio de Janeiro, Brazil

and Defense Engineering Graduate Program

Military Engineering Institute

Praça General Tibúrcio 80, Rio de Janeiro, Brazil

E-mail: fisica.dna@gmail.com

Received: 10 November 2018

Accepted: 4 September 2019

\section{Introduction}

This work aims to help in decision-making process and development of response method regarding a large-scale radionuclide release by a radiological dispersal device (RDD) and in optimizing cost emerging between health risks and the monetary impact. The HotSpot computer model was used to simulate a hypothetical scenario and calculate lung absorbed doses, which in turn are used in the BEIR $\mathrm{V}$ recommended model to estimate excess relative risk (ERR) of lung cancer among the potentially affected population $[1,2]$. This forms the basis for proposing immediate protective actions.

The modern world uses nuclear energy in many steps of its productive chain of goods and services, ranging from quality assurance in industrial production to medicine and energy generation in nuclear power plants. This large-scale use of the nuclear technology requires a high level of safety. Complex scenarios ensue from the release of radioactive material into the environment that might lead to massive environmental exposure, severely affecting a large number of individuals who would have to be evacuated from the risk (affected) zone. Accomplishing a quick and orderly evacuation would be an intricate and highly complex task that would require prompt action of well-prepared rescue teams and experts under the supervision of highly capable decision-making authorities. The core purpose of 
this work is to study such a scenario by applying (a) a methodology to deal with radiological emergencies and (b) risk evaluation performed with converging independent resources for supporting decision-making during the early phase. The consequences of intense ionizing radiation regions can include interdiction of large areas where the access can be banned for relatively long periods due to the threat to public health. Typically, such scenarios involve a high degree of initial misinformation about the radiation source, its spatial distribution, as well as the possible paths of environmental transport of these contaminants $[3,4]$.

Usually, emergency response operations are compromised by a lack of initial information about the scenario. Therefore, once the source term is known, a methodology that is capable of providing useful basic information that positively impacts the ability to manage response team safety, operational cost-effectiveness and public health with minimum data available is vital for communicating risks and comparison with the actual data from the scenario.

This work, through the convergence of two tools, helps decision-making in crisis scenarios facing minimal information resources. There is no information given on the geographical area assumed as this work is for a generic area. On the other side, there are quite good and realistic computer models that could be used to assess the information about radiation dose as a consequence of an RDD, such as hazard prediction and assessment capability (HPAC) [5]. Two approaches are considered: (a) risk modeling and consequence evaluation, both to the environment and to the potentially affected population and (b) cost-effectiveness during the initial phase.

A radiological scenario has been modeled in order to evaluate incidence risk of lung cancer to the potentially affected population as a function of time. Data from the simulations provided a first picture of the situation, allowing immediate protective actions to be chosen. Furthermore, an estimate of detrimental financial cost was calculated by using the monetary coefficient and the estimated collective doses [6-8].

\section{Methods}

A simulation of the RDD in action, including the release of americium-241 (Am-241), was made. In the first step, the HotSpot software was used to estimate the absorbed doses due to inhalation. The estimated doses were then inserted into the BEIR V equations for calculation of the ERR and the probability of causation (PC) for lung cancer incidence; this approach is addressed as convergence methodology [3].

The HotSpot Health Physics Code, version 3.0.3, developed by the American laboratory Lawrence Livermore National Laboratory (LLNL), is freely accessible and free of charge. It is often used also by the International Atomic Energy Agency (IAEA). It was designed as a fast and portable computational tool in order to provide and to evaluate incidents involving radioactive materials. HotSpot is based on a conservative model to estimate the time- and space-dependent concentration of radionuclides and consequent radiation dose from release of radioactive materials into the atmosphere [9]. The code uses a Gaussian model to calculate the dispersion of the radioactive material as it is carried by the wind over the impacted area, depending mainly on the weather conditions, characterized by the classes of Pasquill-Gifford [9].

HotSpot simulates the scenario based on the Gaussian plume model, which is not particularly accurate in urban settings; the code outputs a conservative picture that is convenient for a first-order approach. Considering a more realistic model and comparisons with the Gaussian model, Harper and colleagues [10] discussed likely distribution of radioactivity from an RDD-type event and many differences between releases in urban scenarios.

HotSpot generates an extensive set of data and unsophisticated graphical interpretations of outputted data. Thus, it greatly simplifies the description of the event. The software considers the following parameters: (a) atmospheric dispersion model, (b) meteorological conditions (wind speed, stability class, and height of the inversion layer), (c) nature of the release, (d) rate of deposition, (e) explosion altitude, (f) explosion energy, and (e) sample and exposure time. Data provided include (a) total effective dose equivalent (TEDE), which corresponds to all doses of all the routes that the individual was exposed to, (b) equivalent dose absorbed in the lungs, (c) ground deposition contours, and (d) gamma ground shine contribution. For the simulations, the following characteristics, shown in Table 1, were considered in this work. Table 1 shows the input data for HotSpot to perform the scenario simulation.

In an RDD scenario, it is likely that much of the radioactivity will be in a particulate form that will deposit near the explosion site (first few kilometers). The fraction of breathable particles is a key parameter in assessing dose to the lungs, and this information was considered by HotSpot code.

The epidemiological model BEIR V [11] provides means of estimating ERR and PC impairment for whole-body external exposure to photons (e.g. gamma rays). The ERR, used primarily by radiation epidemiologists [12], is of peculiar interest. ERR represents the excess risk per unit of exposure divided by the background risk [13]. PC, which is the risk of a given cancer attributable to radiation exposure divided by the total risk for that cancer, was determined from the statistical correlation between the occurrence of morbidity and exposure to ionizing radiation. Table 2 shows the equations used to calculate ERR (Eqs. (1) and (2)) for lung cancer incidence, PC (Eq. (3)) [9], and the detrimental cost equation (Eq. (4)), where $r_{0}(a, s)$ is the baseline rate taken as unit for simplification purposes, $a$ is the attained age, $s$ is the sex, $t$ is the time since exposure (years), $\alpha_{1}=0.636 \mathrm{~Sv}^{-1}, \beta_{1}=-1.437, \beta_{2}=0.711$, $D$ is the dose (in Sv), and the risk is measured by ERR/Sv. The parameters $\beta_{1}$ and $\beta_{2}$ are the coefficients for the time after the exposure for male and 
Table 1. Main input data for HotSpot

\section{Material}

Material at risk (MAR)

Total effective equivalent dose (TEDE)

Damage ratio (DR) fraction of the MAR that is actually impacted in the scenario

Fraction breathable $(\mathrm{RF})$ - fraction of aerosol material that is breathable (aerodynamic diameter $(\mathrm{AD}) \leq 10$ microns)

Filtration factor (LPF) is the fraction of MAR, which goes through some confinement or filtration mechanism. For free-release scenarios, the leak path factor is 1 by default

Breathable material $=\mathrm{MAR} \times \mathrm{DR} \times \mathrm{LPF} \times \mathrm{ARF} \times \mathrm{RF}$

Non-breathable material $=$ MAR $\times$ DR $\times$ LPF $\times$ ARF $\times(1-R F)$

Wind speed $(h=10 \mathrm{~m})$

Explosive

Terrain
Am-241

7.4000E+11 Bq

TEDE $=$ inhalation + submersion

+ soil deposition + resuspension

$1.000(100 \%)$

$0.200(20 \%)$

1.000

$1.48 \mathrm{E}+11 \mathrm{~Bq}$

$5.92 \mathrm{E}+11 \mathrm{~Bq}$

$5.00 \mathrm{~m} / \mathrm{s}$

10.00 pounds of TNT $(25 \mathrm{~kg})$

Standard - produces the most conservative estimates
Table 2. Equations used to calculate ERR (Eqs. (1) and (2)) for incidence of lung cancer, PC (Eq. (3)), and estimated cost of detriment (Eq. (4))

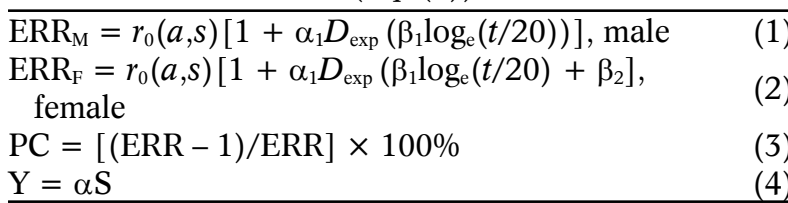

female, respectively. The parameter $\beta_{2}$ (female) adjusts the ERR experimental curve as the relative risk decrease is gender dependent [11].

The form of the model (in particular, the time trend) was based on the observed patterns of lung cancer incidence in the UK and also on spondylitis data [14]. Some characteristics, such as individual health condition, might also increase radiosensitivity. It seems that age and gender differences may result in changes in the radiation exposure effects with increased risk for children, the elderly and women [15]. Additionally, International Commission on Radiological Protection (ICRP) Publication 103 states that women are approximately 35 percent more susceptible to radiation exposure than men [16]. $\mathrm{Y}$ is the detriment cost, $\mathrm{S}$ is the collective dose, and $\alpha$ is the monetary factor expressed by (US\$/individual) $\times$ sievert.

The collective dose is the sum of the individual doses received in a given period by a specified population from exposure to a specified source of radiation. The collective dose is expressed in men-Sv. Since the 1980s, in quantitative optimization evaluations, the value of the monetary coefficient per unit of collective dose should not be lower than the national currency equivalent to US\$ 10000.00 per unit of collective dose per individual [6-8].

The concept of detrimental cost is defined as the cost of injury obtained per unit dose received by each individual or the total health damage experienced by an exposed group of individuals and their offspring as a result of exposure to a radiation source. It is a multidimensional concept. Its main components are stochastic variables such as probability of fatal or nonfatal cancer, hereditary effects, and potential death [17], and its value is obtained by means of Eq. (4).

The ICRP Publication 22 suggests that it would be useful to express the collective dose assessment in monetary units. Thus, the advantage in reducing the collective dose can be compared directly against the cost needed to produce such reduction. Thus, the best optimization solution is the one that yields the lowest cost of total detriment. Examples of value adopted by the ICRP and some countries are as follows: (a) developed world: US\$20 000.00, (b) Japan: US\$ 25 000.00, and (c) Brazil: US\$ 10000.00 [18].

\section{Results}

The equivalent dose absorbed in the lung is represented as a function of distance from the release site as shown in Fig. 1. According to the adopted

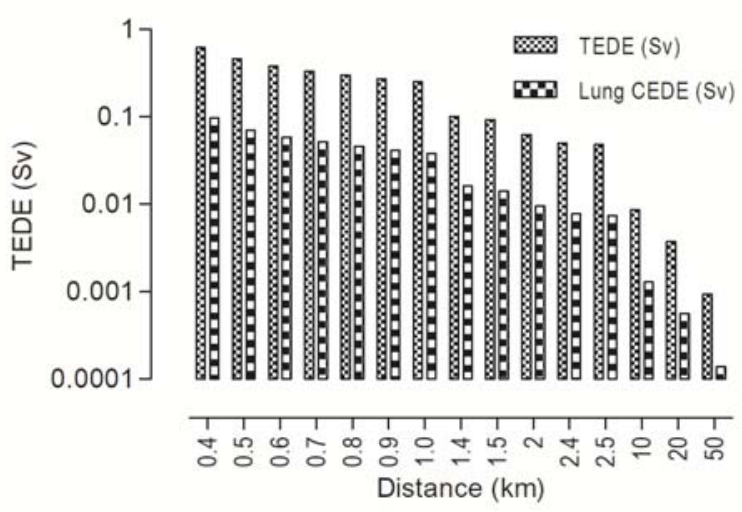

Fig. 1. TEDE and CEDE for lungs as a function of distance from the release site. 

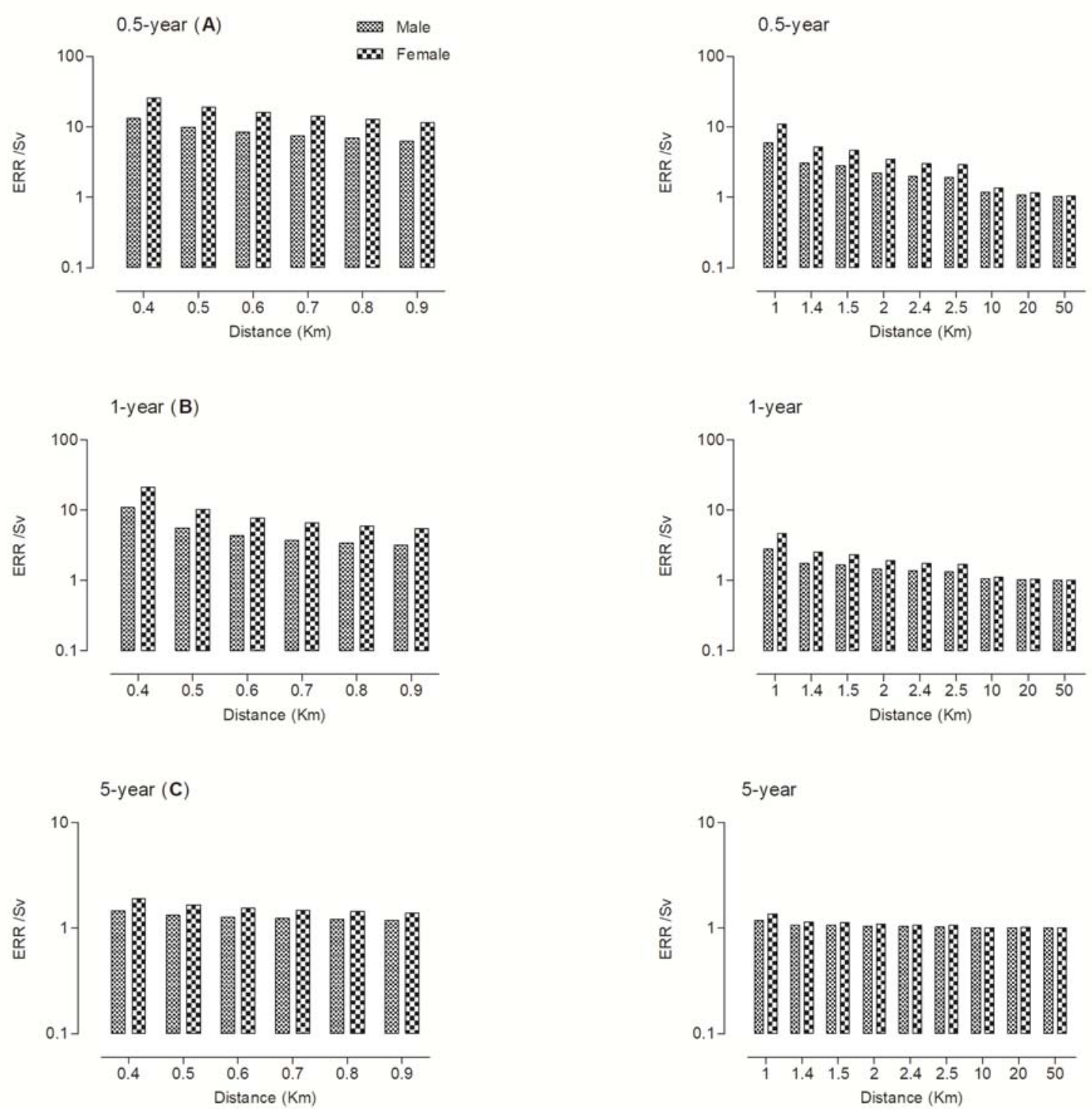

Fig. 2. Excess relative risk (ERR) calculations as a function of time for (A) 0.5 , (B) 1 , and (C) 5 years since exposure.
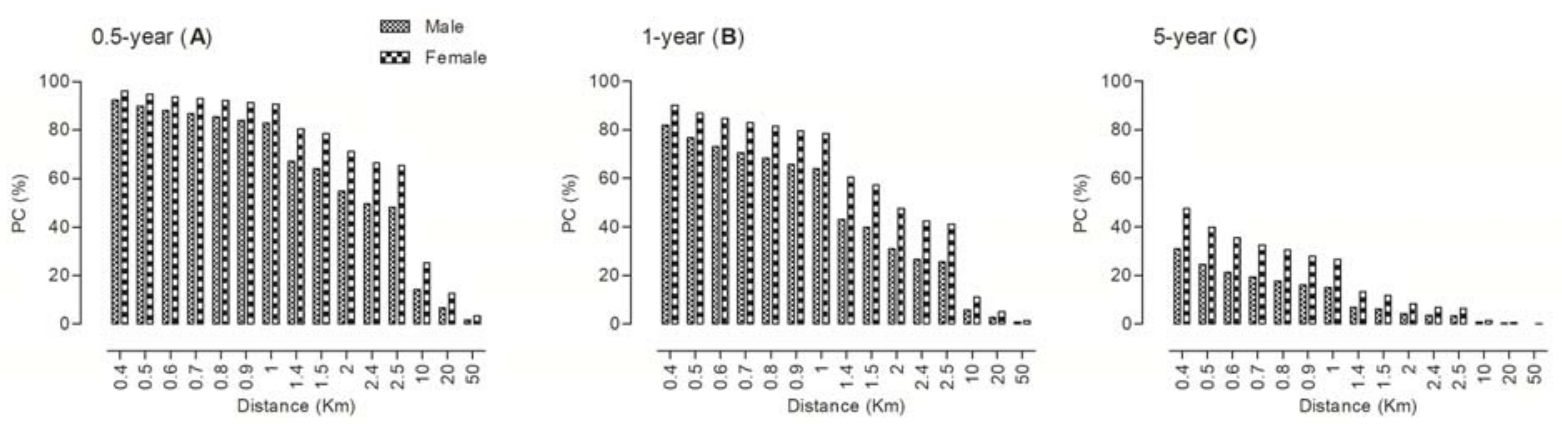

Fig. 3. Probability of causation (PC) calculations for: (A) 0.5, (B) 1, and (C) 5 years since exposure.

scale, the first distance $0.4 \mathrm{~km}$ was closest to that calculated by HotSpot, which is the limit where no mechanical or thermal effects from the explosive blast are felt and so radiation is the only effect. Although the dose in this region as being from $1 \mathrm{mSv}$ to $50 \mathrm{mSv}$ seems excessive, the main feature for the first-order approach is to be conservative.

Figure 2 shows the ERR estimate considering radiation dose and sex of the adult population po- tentially affected as a function of time and distance from the release point.

Figure 3 shows the calculated PC results as a function of distance and time elapsed since the event. Considering that the development of the morbidity occurs over longer periods, it is convenient to present the evolution of PC from 5 to 30 years.

Table 3 presents the major results from the simulations and mathematical models applied (see Table 2). 
Table 3. Major results from simulations based on equations listed in Table 2. Lethal dose $\left(\mathrm{LD}_{50 / 30}\right)$ is the radiation dose that causes $50 \%$ of the exposed population to die within 30 days following the exposure

\begin{tabular}{|c|c|c|c|c|}
\hline Location (L) & Individuals & Collective dose $[\mathrm{Sv}]$ & Cost detriment [US\$] & TEDE $[\mathrm{mSv}]$ \\
\hline $\begin{array}{l}9.6 \mathrm{~Sv}(10 \mathrm{~m}) \\
\approx 4 \mathrm{~Sv}(30 \mathrm{~m})\end{array}$ & \multicolumn{4}{|c|}{ Dose $\geq \mathrm{LD}_{50 / 30}\left(4 \times 10^{3} \mathrm{mSv}\right)$} \\
\hline $1.5 \mathrm{~Sv}(274 \mathrm{~m})$ & \multicolumn{4}{|c|}{ Radiation and/or injuries from blasting (combined injuries) } \\
\hline $274 \mathrm{~m}<\mathrm{L}<1.4 \mathrm{~km}$ & 5485 & $3.08 \mathrm{E}+03$ & 30768830.40 & \\
\hline $1.4 \mathrm{~km}<\mathrm{L}<2.4 \mathrm{~km}$ & 14691 & $9.99 \mathrm{E}+02$ & 9989880.00 & $50<D<100$ \\
\hline $\begin{array}{l}2.4 \mathrm{~km}<\mathrm{L}<50 \mathrm{~km} \\
\mathrm{~L}>50 \mathrm{~km}\end{array}$ & \multicolumn{3}{|c|}{ Background } & $1 \leq D \leq 50$ \\
\hline
\end{tabular}

The number of inhabitants was estimated considering the local population density at downtown in working days as 10000 inhabitants $/ \mathrm{km}^{2}$ in rush hour. Even though such population density might impose bias overestimating the results, it should be considered as a pessimist foreseeing to serve as basis for decision.

\section{Discussion}

The results show that the explosion of an RDD device results in an increased equivalent effective total dose in the lungs of individuals up to a distance $50 \mathrm{~km}$ from the RDD triggering site. After this point, the radiation levels are so reduced that they can be considered negligible and only normal background radiation is present. The estimated value of the equivalent dose in the lungs was used to estimate the ERR for lung cancer as a function of time. The ERR was estimated for a time interval from 6 months to 30 years after exposure to the radiation field, considering only adults in the potentially affected population. It should be taken into account that the simulation is conservative. In a real urban setting, most people will be inside the buildings when the event takes place. A large building can reduce exposure by a factor of 20 [19]. Even for smaller buildings, if individuals shelter inside until the plume passes and then goes outside, the exposure will be substantially less than predicted by HotSpot codes, which assume everyone is outside in the open space.

Results from Fig. 1 suggest that radiation effects are also strongly dependent on the exact location (distance) of the individual in the RDD scenario with impact on the absorbed dose. Depending on the dose levels, the scenario unfolds in two zones of risk evaluation: (a) an inner region in which the ability of emergency responders could be severely constrained as they will be exposed to ground shine from deposition of particulate matter, which will impact their response and (b) an outer region where long-term public health can be affected. The first zone runs from the RDD release site up to approximately $1 \mathrm{~km}$ away downwind, where the dose remains above $100 \mathrm{mSv}$, which is used in this work as the upper limit for emergency operations. A subregion from the release point up to approximately $0.3 \mathrm{~km}$ is complex, and responders should consider that individuals located in this area would face higher radiation exposure combined with mechanical and thermal impacts from the explosion. From the release site to $0.4 \mathrm{~km}$, there is a rapid decrease in ERR, while from $0.4 \mathrm{~km}$ to $0.9 \mathrm{~km}$, the dose re- duction is relatively small. Across this first zone out to $1 \mathrm{~km}$, responders should include the possibility of immediate biological effects occurrence such as acute radiation syndrome, which is extensively described by Mettler [20]. Within this $1 \mathrm{~km}$ zone, females are significantly more likely (up to twice as likely) to get a lung cancer within ten years of the exposure. The male and female incidences of cancer become similar over longer time periods of about 5 years following the exposure.

In the second zone beyond $1 \mathrm{~km}$, the dose is estimated below the $100 \mathrm{mSv}$ emergency responder limit. Data shown in Fig. 1 for individuals beyond $1 \mathrm{~km}$ downwind still show some elevated level of ERR near term, especially for females. This finding may suggest that it may be difficult to check for new cases of lung cancer later on among those located beyond $1 \mathrm{~km}$, not only because of risen risk but also because of poor statistics normally faced in such scenarios. This might influence positively the cost-effectiveness level.

The data calculated for PC presented in Fig. 3 also suggest that female individuals are under a higher chance of correlation between the development of morbidity and the exposure when compared to male ones. This trend points to the strong dependency of time, with the differences between genders decreasing with time elapsed since exposure. Time negatively affects the correlation between the development of the morbidity and the previous radiological exposure.

Table 3 presents, in a concise form, data that could be used in and support a decision-making. The data presented are as follows: (a) size of potentially affected population, (b) collective dose, (c) approximate detriment cost, and (d) total effective equivalent dose. Such data give an overview of the situation and offer the opportunity to streamline the logistics activities and to allocate resources. Results presented in Table 3 suggest that there is a potential increasing demand of such resources as the distance to which response and recovery measures from the RDD release are to be implemented. This is dominated by the increasing size of the population accounted for.

Considering radiation dose distribution within the plume, protective measures may have a positive financial impact. In such situations, prompt and precise decisions are necessary and may include urgent measures such as (a) sheltering and (b) evacuation. These measures are supported by an assessment of the external exposure conditions and inhalation doses of the population $[21,22]$. 
The data generated from simulation and calculations, which are shown in Table 3, can contribute to and support the planning of countermeasures as they provide a brief overview of the scale considering the efforts to be made in order to provide evacuation and sheltering.

Additionally, the cost of detriment is dependent on the location of individuals. Table 3 lists estimates of financial resources to be allocated for the scenario into two categories, depending on the radiation dose, regardless of accounting the cost of emergency response, or decontamination: (a) $50 \mathrm{mSv}<D<100 \mathrm{mSv}$ ( $\approx$ US $\$ 40 \mathrm{Mi}$ ) and (b) $1 \mathrm{mSv}<D \leq 50 \mathrm{mSv}$ (US\$ $500 \mathrm{Mi}$ ). For these ranges, in accordance with international or local standards, the allocation of resources' assessment is performed in order to determine the cost amount to be destined.

\section{Conclusion}

This work has demonstrated that a typical RDD event can lead to serious adverse effects to a population. In addition, the results suggest that scientific information promptly obtained through convergence methodology requiring limited input data may produce a positive impact regarding cost and emergency planning activities when supporting the decision-making process. In this way, the proposed methodology offers usable data that could facilitate the decision-making process during such emergencies by promptly providing some important quantitative aspects.

Such aspects can be (a) an estimate of the potentially affected population size; (b) an overview of the scenario that would help choose the best protection measures; (c) a prioritization of activities of responders and evacuees according to gender; (d) an estimate of TEDE and committed effective dose equivalent (CEDE) doses, which are the core variables; (e) generate a matrix of incidence for lung cancer within a period of 30 years; and (f) estimate costs of detriment, considering the initial conditions and the operational capacity of authorities for reallocation of resources when necessary.

The potentially affected population is subject to exposure, and resources should be properly managed over time in support of both the attendance to potential developments of morbidities related to the event. Consequently, in this way, the results from this work might contribute by advising the authorities to seek for further resources in the long-term perspective.

Acknowledgments. The authors wish to thank the colleagues who contributed with many useful comments and suggestions. They also thank the Forensic Institute at Cranfield University and the Defense Academy of the United Kingdom for the scientific support. This work was supported by the Brazilian National Council for Scientific and Technological Development $(\mathrm{CNPq}$ Grant No. 409622/2016-8).

\section{ORCID}

Edson R. Andrade (Dhttp://orcid.org/0000-0001-8800-6105

\section{References}

1. Maillie, H. D., \& Jacobson, A. P. (1992). A graphical method of estimating fatal radiation-induced cancers using the BEIR V method. Health Phys., 63(3), 273-280.

2. Maillie, H. D., Simon, W., Watts, R. J., \& Quinn, B. R. (1993). Determining person-years of life lost using the BEIR V method. Health Phys., 64(5), 461-466.

3. Rother, F. C., Rebello, W. F., Healy, M. J., Silva, M. M., Cabral, P. A., Vital, H. C., \& Andrade, E. R. (2016). Radiological risk assessment by convergence methodology model in RDD scenarios. Risk Anal., 36(11), 2039-2046. DOI: 10.1111/risa.12557.

4. Andrade, C. P. S., Souza, C. J., Camerini, E. S. N., Alves, I. S., Vital, H. C., Healy, M. J. F., \& Andrade, E. R. (2018). Support to triage and public risk perception considering long-term response to a Cs-137 radiological dispersive device scenario. Toxicol. Ind. Health, 34(6), 433-438. https://doi. org/10.1177/0748233718762920.

5. Purves, M., \& Parkes, D. (2016). Validation of the DIFFAL, HPAC and HotSpot dispersion models using the Full-Scale Radiological Dispersal Device (FSRDD) field trials witness plate deposition dataset. Health Phys., 110(5), 481-490.

6. Thomson, W. H., \& Roberts, P. J. (1986). Cost-benefit analysis in radiation protection. Nucl. Med. Commun., 7(12), 855-856.

7. Weatherburn, H. (1984). A realistic approach to costbenefit analysis in radiation protection. Br. J. Radiol., 57(681), 847-848. https://doi.org/10.1259/00071285-57-681-847.

8. International Commission on Radiological Protection. (1983). Cost-benefit analysis in the optimization of radiation protection. Ann. ICRP, 10(2/3). (ICRP Publication 37).

9. Homann, S. G. (2013). HotSpot Health Physics Codes Version 3.0 User's Guide. Lawrence Livermore National Laboratory, CA, USA.

10. Harper, F. T., Musolino, S. V., \& Wente, W. B. (2007). Realistic radiological dispersal device hazard boundaries and ramifications for early consequence management decisions. Health Phys., 93(1), 1-16.

11. International Atomic Energy Agency. (1996). Methods for estimating the probability of cancer from occupational radiation exposure. Vienna: IAEA. (IAEATECDOC-870).

12. Preston, D. L., Ron, E., Tokuoka, S., Funamoto, S., Nishi, N., Soda, M., Mabuchi, K., \& Kodama, K. (2007). Solid cancer incidence in atomic bomb survivors: 1958-1998. Radiat. Res., 168(1), 1-64. https:// doi.org/10.1667/RR0763.1.

13. Lee, W. C. (2014). Excess relative risk as an effect measure in case-control studies of rare diseases. PLoS One, 10(4), e0121141. https://doi.org/10.1371/ journal.pone.0121141. 
14. Darby, S. C., Doll, R., Gill, S. K., \& Smith, P. G. (1987). Long term mortality after a single treatment course with X-rays in patients treated for ankylosing spondylitis. Br. J. Cancer, 55(2), 179-190. https:// doi.org/10.1038/bjc.1987.35.

15. Narendran, N., Luzhna, L., \& Kovalchuk, O. (2019). Sex difference of radiation response in occupational and accidental exposure. Front. Genet., 10, 260. https://doi.org/10.3389/fgene.2019.00260.

16. International Commission on Radiological Protection. (2007). The 2007 Recommendations of the International Commission on Radiological Protection. Ann. ICRP, 37(2/4), 1-332. (ICRP Publication 103).

17. International Commission on Radiological Protection. (1989). Optimization and decision-making in radiological protection. A report of a Task Group of Committee 4 of the International Commission on Radiological Protection. Ann. ICRP, 20(1), 1-60.

18. International Commission on Radiological Protection. (1973). Implications of Commission recommenda- tions that doses be kept as low as readily achievable. (ICRP Publication 22). Oxford: Pergamon Press.

19. Dillon, M., Kane, J., Nasstrom, J., Homann, S., \& Pobanz, B. (2016). Summary of building protection factor studies for external exposure to ionizing radiation. Lawrence Livermore National Laboratory, CA, USA. (LLNL-TR-684121).

20. Mettler, F. A. Jr. (2005). Medical resources and requirements for responding to radiological terrorism. Health Phys., 89(5), 488-493.

21. Conklin, C., \& Edwards, J. (2000). Selection of protective action guides for nuclear incidents. J. Hazard. Mater., 75(2/3), 131-144. https://doi.org/10.1016/ S0304-3894(00)00176-X.

22. Sorensen, J. H., Shumpert, B. L., \& Vogt, B. M. (2004). Planning for protective action decision making: evacuate or shelter-in-place. J. Hazard. Mater., 109(1/3), 1-11. https://doi.org/10.1016/j.jhazmat.2004.03.004. 